\title{
Gyrokinetic Prediction of Microtearing Turbulence in Standard Tokamaks
}

\author{
H. Doerk, F. Jenko, T. Görler, D. Told, M. J. Pueschel, and D. R. Hatch \\ Max-Planck-Institut für Plasmaphysik, EURATOM Association, \\ Boltzmannstraße 2, D-85748 Garching, Germany
}

\begin{abstract}
First global gyrokinetic simulations of microtearing instabilities in ASDEX Upgrade geometry provide increasing evidence for the existence of these modes in standard tokamaks. It is found that even in only moderately large devices, nonlocal effects like profile shearing are negligible, supporting the use of an efficient flux-tube approach. Nonlinear gyrokinetic simulations show that the resulting level of magnetic electron heat flux can be experimentally relevant.
\end{abstract}

\section{INTRODUCTION AND BACKGROUND}

In burning ITER [1] plasmas, the fusion processes will predominantly heat the electrons. It is thus important to understand and be able to predict electron thermal transport via microturbulence in magnetically confined plasmas. In this context, many key aspects are still under investigation, and the overall picture continues to evolve. Prominent examples include the contributions of sub-iongyroradius scale turbulence driven by electron temperature gradient (ETG) modes [2] and the role of magnetic stochasticity caused by small-scale reconnecting modes. The latter question has recently been addressed anew by means of high-resolution gyrokinetic turbulence simulations [3-6], building on fairly recent hardware and software advances. These modern methods allow to follow up on theoretical considerations which started already decades ago.

In 1973, Stix argued that even minute magnetic perturbations can greatly enhance the electron thermal transport if these perturbations are resonant. [7] Microinstabilities are a possible source of such perturbations, provided that they exhibit a resonant component of the vector potential, $\tilde{A}_{1 \|}$. The magnitude of this resonant component, which is essentially an integral of $\tilde{A}_{1 \|}$ along the field line, crucially depends on the parity of the perturbation. In an up-down symmetric tokamak, eigenmodes (with a vanishing radial wavenumber $k_{x}$ ) are characterized by either tearing (even) or ballooning (odd) parity with respect to $\tilde{A}_{1 \|}$. Importantly, the well-known ion temperature gradient (ITG) modes and trapped electron modes (TEM), at $k_{x}=0$, belong to the second category. The corresponding field-line integral vanishes and thus cannot directly break field lines. Magnetic stochasticity can be expected, however, when tearing-parity modes, such as the microtearing mode, are present and create small-scale magnetic islands which may overlap to create a stochastic field. In such a stochastic field, the fairly large parallel heat conductivity is coupled to the cross-field component, which may greatly enhance electron heat transport. First results in this area have aleady been obtained in the 1970's by several authors. [8-11] Moreover, it has recently been proposed that in finite $\beta$ ITG/TEM turbulence, linearly stable microtearing modes can be excited via a nonlinear coupling to zonal modes.[5] Interestingly, this can explain both the occurrence of stochastic fields [6] and the quadratic scaling of the magnetic transport which contradicts standard quasilinear transport models.[12]

In the present work, we will focus on linearly unstable microtearing modes. These are the gyrokinetic analogues of the well-known magnetohydrodynamic (MHD) tearing modes, although they involve quite different physics. While the MHD tearing modes gain free energy by relaxing the magnetic field to a lower-energy state, microtearing modes draw free energy from the background electron temperature gradient. Also, a certain level of collisionality is required to allow for magnetic reconnection via the formation of a narrow current layer about a mode rational flux surface. Drake and co-workers [9] contributed significantly to the theoretical understanding of microtearing modes by providing an intuitive picture for the instability mechanism in three regimes named collisionless $\left(\nu_{e i} /|\omega| \ll 1\right)$, semi-collisional $\left(\nu_{e i} /|\omega| \gg 1\right.$ and $\left.\Delta / \rho_{i} \ll 1\right)$ and collisional $\left(\nu_{e i} /|\omega| \gg 1\right.$ and $\left.\Delta / \rho_{i} \gg 1\right)$. Here, $\nu_{e i}$ denotes the electron-ion collision rate, $\omega$ is a typical mode frequency, $\Delta$ is the width of the current layer and $\rho_{i}$ the ion gyroradius. Modern tokamaks, characterized by high temperatures, mostly operate in the semicollisional and collisionless regimes.

A review of the existing literature leads to the conclusion that (even) the linear physics of the microtearing mode is surprisingly complicated. Two destabilizing effects for the electron temperature gradient drive have been reported. One of them is the time-dependent thermal force $[8,9,13-16]$, the second is the influence of the collisionality at the trapped-passing boundary $[10,17,18]$. Moreover, a destabilizing contribution of the electrostatic potential to the parallel electric field inside the current layer has been found in semi-analytic work.[16] A stabilizing effect due to the electrostatic potential is the short-circuiting of the current, thereby limiting the current layer width in the collisional regime. Additional damping mechanisms are the magnetic energy term and the collisional broadening of the Landau resonance.[18] However, it is not entirely clear from the published analytical work whether stabilizing or destabilizing terms dominate under realistic experimental conditions.

In the last decade, linear gyrokinetic studies have been undertaken, indicating a role of microtearing modes first in spherical (small aspect ratio) [19-21] and later also in standard (medium aspect ratio) [22-24] tokamak plas- 
mas. Among other things, these gyrokinetic computations revealed another drive mechanism related to magnetic curvature (thus not accessible in early slab models) which adds to the complexity of the linear mode.[22] Exploring various physical parameter regimes, different types of microtearing modes have been identified. In spherical tokamaks, operating at high $\beta_{e}$, they exist at rather high perpendicular mode numbers of $k_{y} \rho_{i} \sim$ 1 , whereas in standard tokamaks, one typically finds $k_{y} \rho_{i} \sim 0.1$. A quite extreme difference in the radial scales between fine-scale parallel electron current $\left(\tilde{j}_{\| e}\right)$ as well as electrostatic potential $(\tilde{\phi})$ structures and largescale $\tilde{A}_{\|}$perturbations is commonly seen, an effect which makes it computationally expensive to compute even linear modes.

To predict the resulting level of turbulent electron heat transport, however, it is required to transcend linear theory. A semi-analytic transport prediction of Drake and co-workers has successfully been used to interpret the results of a rather collisional discharge in the National Spherical Torus Experiment (NSTX).[25, 26] However, such analytical treatments of the nonlinear microtearing problem suffer from severe simplifications. Significant progress in this regard has been made only within the last year or so, when investigations of the nonlinear dynamics of microtearing modes by means of ab initio gyrokinetic simulations have been reported in Ref. [3] for standard tokamaks and in Ref. [4] for spherical tokamaks. In both device types, the turbulence level and thermal transport is greatly enhanced with increasing electron temperature gradient. A series of simulations performed for a certain NSTX discharge reproduces the experimentally determined favorable scaling with collisionality. [27] An experimentally relevant level of heat flux is obtained in these nonlinear gyrokinetic runs, establishing linearly unstable microtearing modes as an additional candidate to explain anomalous electron heat transport.

It should be pointed out that these simulations are extremely demanding in terms of both the required computational resources and the level of detail in the physics model. For example, in the radial direction, high resolution and large domain size are needed, because of the aforementioned multi-scale features in $\tilde{A}_{\|}$and $\tilde{\phi}$. For this reason, computations could so far only be obtained under certain simplifications. For standard tokamak simulations, a geometry model assuming concentric circular flux surfaces (without Shafranov shift) has been used to date, and studies using realistic geometry are obviously the next step. Furthermore, up to now, all nonlinear computations have been performed in the local (fluxtube) limit, neglecting radial dependencies of quantities like temperature, density, their gradients, and magnetic geometry. The local approximation is expected to hold when the tokamak minor radius $a$ becomes much larger than the gyroradius $\rho_{i}$. While finite machine size effects usually stabilize modes like the ITG instability due to profile shearing, [28-30] it is a priori not clear how microtearing modes are affected. In the present work, we relax these constraints in the context of linear and nonlinear gyrokinetic simulations, employing realistic ASDEX Upgrade (AUG) parameters.

To this aim, we use the GENE code [2, 29, 31] which solves the nonlinear gyrokinetic Vlasov-Maxwell system self-consistently on a fixed grid in phase space. GENE is physically comprehensive and thus well suited to study a problem as complex as microtearing turbulence. Parallel and perpendicular magnetic fluctuations as well as external $E \times B$ shear flows are allowed, and a LandauBoltzmann collision operator is implemented which includes energy and momentum conserving terms.[31] Interfaces to MHD equilibrium codes are also provided.[32] Radial profiles of temperature, density, and magnetic geometry can be included in GENE, but the code can also be run in the local (flux-tube) limit, taking advantage of spectral methods for radial derivatives. Most of these features are important in the course of this work.

The paper is structured as follows. In Sections II and III, global GENE results on microtearing modes are presented, and a system size scan of linear microtearing modes is performed. Since it turns out than even for relatively small machines, the local limit is adequate, the flux-tube version of GENE is used in the following. In Section IV, the AUG discharge 26459 is studied with respect to critical electron temperature gradient and $\beta_{e}$. The two following Sections are devoted to two important aspects of the linear instability, which are the influence of collisions (Section V) and the characteristics of the current layer at the mode rational surface (Section VI). Finally, in Section VII, we summarize microtearing turbulence results, adding a detailed analysis of the properties of electron heat transport that is related to magnetic stochasticity.

\section{GLOBAL LINEAR MICROTEARING MODES IN ASDEX UPGRADE}

To begin with, we perform a linear stability analysis of the AUG shot 26459 with Gene. This well diagnosed H-Mode discharge shows Type II ELMs (Edge Localized Modes) and is characterized by strong plasma shaping and high density, with high triangularity, high elongation, and high $q_{95}$. Discharges where Type II ELMs are created start as standard H-Modes with Type I ELMs and are then pushed upwards to a near doublenull configuration. $[33,34]$ When the plasma density is sufficiently high, Type I ELMs develop into the faster and weaker Type II ELMs. Additionally, the electron temperature decreases in the outer core region. Due to the high density, the collisionality is rather large, which is why one might expect tearing modes to appear. However, we find $\nu_{e i} /|\omega|<10$, and thus the collisional regime for tearing modes defined in [9] is not reached.

The results presented below correspond to the Type II ELM phase. Temperature and density profiles as well as magnetic geometry are averaged over a timeslot be- 
FIG. 1: (Color online) Temperature and density profile fits and measured data points from the AUG data base for discharge 26459 in the time interval between 4.0 and 4.6 seconds. We restrict ourselves to $\rho_{\text {pol }}>0.4\left(\rho_{\text {tor }}>0.29\right)$ to obtain a more accurate fit in the outer region. The interferometer density measurement (black squares) is well matched when one integrates the density fit along the corresponding line of sight (red crosses).

tween 4.0 and 4.6 seconds of that discharge. The measured data for temperature and density are taken from the AUG database (see Fig.1). The resulting pressure gradient in the plasma edge, together with the signals of the magnetic pickup coils, serves as input boundary conditions for the ClisTE code that reconstructs the equilibrium. The resulting safety factor profile is depicted in Fig. 3. The field-aligned coordinate system used in GEnE is then obtained with the TRACER module. In this work, $\rho_{\text {tor }}=x / a$ is used as a radial coordinate, where $a=\sqrt{\left(\Psi_{\text {tor }, \text { sep }} /\left(\pi B_{0}\right)\right)}$ is the effective minor radius, such that the separatrix is located at $\rho_{\text {tor }}=x / a=1$.

Using these profiles as input, we perform global linear GENE simulations of microtearing modes in toroidal geometry. Fig. 2 shows the $\tilde{A}_{\|}$and $\tilde{\phi}$ contour plots for $n=$ 11 perturbations. For the most unstable microtearing mode, one can identify the dominant poloidal mode number $m=24$. The electrostatic potential perturbation and the parallel electron current shown in Fig. 3 are rather localized about the corresponding mode rational surface of $q=24 / 11$, while the $\tilde{A}_{\|}$perturbation is more extended. The plasma parameters at this position $\left(\rho_{\text {tor }}=0.6\right)$ are: $\beta_{e}=0.00423, \nu_{e i}=0.685 c_{s} / a, q=2.18 \approx 24 / 11$, $\hat{s}=1.31, a / L_{T e}=3.02, a / L_{n}=0.376, a / L_{T i}=2.185$,

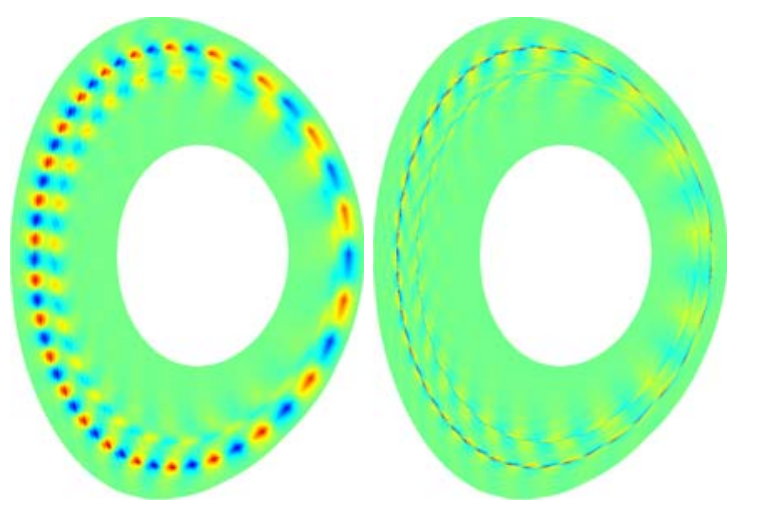

FIG. 2: Contours of $\tilde{A}_{\|}$and $\tilde{\phi}$ in the poloidal plane for $n=$ 11. The most unstable microtearing mode is localized at the $q=24 / 11$ flux surface in this case. The mode rotates in the electron diamagnetic direction. $\tilde{A}_{\|}$forms larger structures, while $\tilde{\phi}$ is closely bound to the mode rational surface.

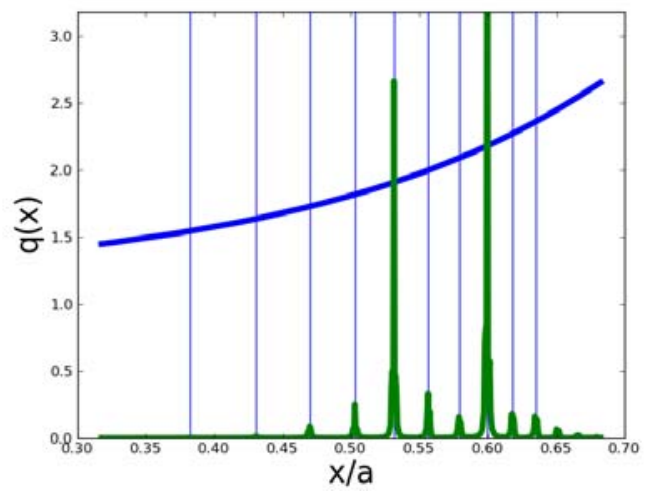

FIG. 3: (Color online) The $q$ profile of AUG discharge 26459 is shown. The electron current perturbations, $\tilde{j}_{\| e}$, peak at the rational flux surfaces for $n=11$. For the chosen time slice, the fastest growing mode $m=24$ is not yet completely dominating.

$T_{i} / T_{e}=1.192$, and $\rho^{*}=0.00304$. Here, we define the electron beta as $\beta_{e}=8 \pi n_{e} T_{e} / B_{0}^{2}$ and the electron-ion collision rate as $\nu_{e i}=4 \pi n_{e} e^{4} \ln \Lambda\left(2 T_{e}\right)^{-3 / 2} m_{e}^{-1 / 2}$. For this particular run, we reduced the ion temperature gradient to $a / L_{T i}=1.6$, which speeds up the computation due to a shorter transient phase. However, local runs have shown that the microtearing instability is indeed the most unstable mode also for the nominal value of $a / L_{T i}$. Convergence studies show that a radial grid spacing of $0.2 \rho_{i}$ is required to obtain the correct growth rate.

\section{SYSTEM SIZE EFFECTS}

Having established that global GENE simulations of actual AUG discharges exhibit microtearing instabilities, a natural follow-up question is to which degree these modes are influenced by nonlocal effects. To address this issue, 
it is useful to perform systematic scans in the parameter $\rho^{*}=\rho_{i} / a$ which sets the ratio of the typical turbulence scale length and the machine minor radius $a$. Of fundamental interest is the convergence behavior in the local limit, $\rho^{*} \rightarrow 0$. For these $\rho^{*}$ variations, we fix both the radial box length in units of $a$ and the grid spacing of $0.2 \rho_{i}$. Therefore the number of radial grid cells increases as $1 / \rho^{*}$, and the simulations become more computationally expensive for smaller $\rho^{*}$. The profile of the electron temperature gradient is peaked at the center of the radial domain $\left(x_{0} / a=0.5\right)$ in all the runs. This is also where we place the $q_{0}=3$ mode rational surface. We thus expect the mode with the maximum growth rate at this position. The binormal wave vector is $k_{y} \rho_{i}=0.12$, which corresponds to the toroidal mode number $n_{0}=\left(x_{0} / a\right) q_{0}^{-1}\left(k_{y} \rho_{i}\right)\left(\rho^{*}\right)^{-1}$. The dependence on $\rho^{*}$ in this relation implies that larger devices involve more unstable mode numbers. For $\rho^{*}=1 / 50$, the mode density is very low, and only up to a few modes fall into the typical low $k_{y}$ regime of linear instability.

Now, Fig. 4 shows that the local result is even valid for relatively large values of $\rho^{*}$. This comes a bit as a surprise, since ITG modes are known to be strongly stabilized with increasing $\rho^{*}$. A possible explanation can be found in the fundamentally different mode structure. One of the stabilizing $\rho^{*}$ effects is the variation of the diamagnetic drift velocity $\omega_{*}$ across the width of typical eddies, which is associated with some intrinsic shearing. When the shearing rate becomes comparable to the growth rate, the ITG mode is stabilized. In a similar fashion, shearing due to zonal flows saturates the ITG instability. One can understand this by noting that a sheared ITG eddy is linearly damped. In contrast to an ITG mode, in which all relevant fluctuating fields have comparable scale length in radial direction, microtearing modes show inherent multiscale features. The vector potential $\tilde{A}_{\|}$is fairly large-scale, but other fields like $\tilde{j}_{\| e}, \tilde{\phi}$, and $\tilde{T}_{e}$ are peaked (within few $\rho_{i}$ ) about the rational flux surfaces. Those narrow structures are apparently less susceptible to radial shearing effects. However, a certain level of $\rho^{*}$ stabilization is seen in cases for which $\omega_{*}$ is still essentially constant across the extremely narrow current layers, indicating that other fields (like $\tilde{A}_{\|}$) also play a role in the instability mechanism. In Fig. 4, we further observe that the limit $\rho^{*} \rightarrow 0$ is correctly captured.

We note in passing that in some cases, two microtearing modes at different radial positions have very similar growth rates, including the one shown in Fig. 4. Thus, long (initial-value) simulations are required to clearly identify the fastest growing mode. We also point out that careful convergence tests have been performed for $\rho^{*}=0.01$, confirming the need for significant computational resources. In particular, it was found that $40 \times 16$ points in $\left(v_{\|}, \mu\right)$ velocity space and 24 points in the fieldline following direction $z$ are required, as well as a radial grid spacing of $0.2 \rho_{i}$. Here, we use a phase space domain characterized by $L_{x} / a=0.6, L_{v_{\|}}=3.28 v_{T j}$, and $L_{\mu}=11 T_{j} / B_{0}$ for plasma species $j$. In practice, up to

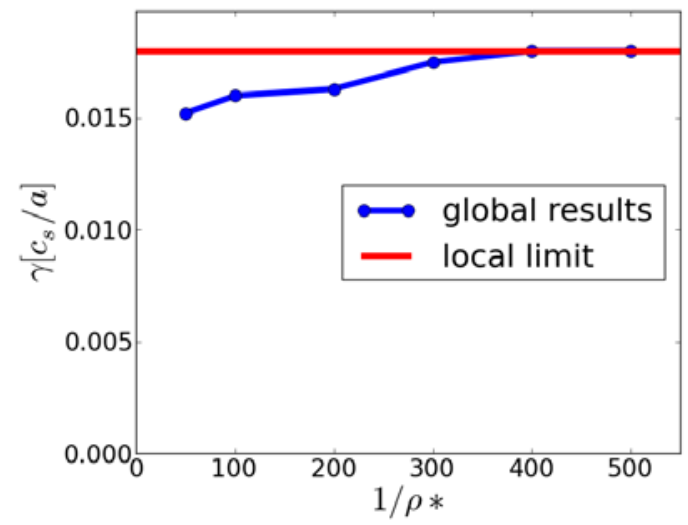

FIG. 4: (Color online) $\rho^{*}$ variation in radially global simulations with fixed gradient profile and binormal wave number $k_{y} \rho_{i}=0.12$. Even at large $\rho^{*}$, profile shear stabilization is moderate, and the local limit $\rho^{*} \rightarrow 0$ is correctly captured.

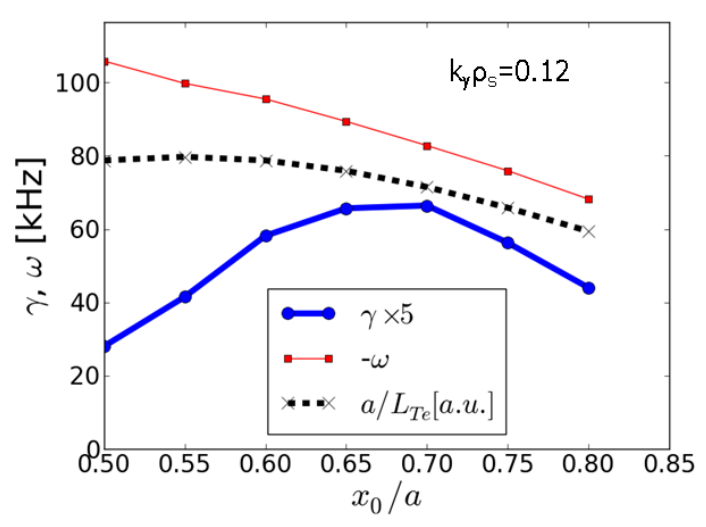

FIG. 5: (Color online) A local scan over the radial position $\rho_{\text {tor }}=x / a$ shows that microtearing modes are unstable over a wide range in the outer half of the tokamak core. For the fixed binormal mode number $k_{y} \rho_{i}=0.12$, the real frequency is around 50 to $100 \mathrm{kHz}$.

about 10,000 CPU-hours are required per linear simulation.

\section{LOCAL SIMULATIONS FOR ASDEX UPGRADE DISCHARGE 26459}

We have verified in the previous section that the (linear) microtearing instability is well represented by the local limit even for moderate values of $\rho^{*}$. AUG has typical $\rho^{*}$ values of a few times $10^{-3}$ and usually falls into that regime. The results presented below are obtained with the local (flux-tube) version of the GENE code. The reason for that is the increased simplicity and computational efficiency of local compared to global computations.

Using local runs, we first determine the linear growth rate as a function of the radial position $\rho_{t o r}$. The physical parameters for these simulations are extracted from 
FIG. 6: (Color online) Microtearing modes dominate the low- $k_{y}$ region. They coexist with an ITG branch at higher wavenumbers. The ETG instability at $k_{y} \rho_{s} \sim 2$ is not shown. When comparing to the results from the AUG database, slightly modified plasma parameters are used, see Tab. I.

exceeded, heat transport due to ITG modes increases rapidly with increasing gradient. The resulting heat flux usually matches the input power (and associated heat flux) at a gradient close to the critical value, implying profile stiffness. This critical gradient, however, experiences a nonlinear up-shift in ITG turbulence. Interestingly, in the analysis of our nonlinear simulations, we will see that a similar effect is expected for microtearing turbulence. Strong transport only sets in once a threshold in the magnetic field fluctuation amplitude (and therefore in the drive strength) is exceeded.

In all gyrokinetic studies to date, a threshold behavior in the parameters $a / L_{T e}$ and $\beta_{e}$ has been observed. Sensitivities on the density gradient seem to depend on the specific parameter regime and magnetic geometry. While NSTX high-k microtearing is damped by $a / L_{n}$ around the experimental value $\left(\eta_{e}=L_{n} / L_{T}\right.$ drive), [35] AUG results have shown a $a / L_{n}$ drive including a threshold [24] or a weak dependence with finite growth rate at $a / L_{n}=0$ [3]. Our study of critical parameters for microtearing thus focusses on $a / L_{T e}$ and $\beta_{e}$, the results are depicted in Fig. 7. The instability threshold for $a / L_{T e}$ is increased for decreasing $\beta_{e}$ in the low-wavenumber limit, but the experimental reference values for both $a / L_{T e}$ and $\beta_{e}$ (Tab. I) are well above this threshold. The critical parameter study performed for our reference AUG dis- 
FIG. 8: (Color online) The dependence of the microtearing growth rate on the collision frequency is moderate. A maximum growth rate is reached at intermediate collision frequencies, where $\nu_{e i} /|\omega| \sim 1-10$, consistent with early slab-geometry calculations $[15,16]$ and gyrokinetic simulations [22]. In ITER baseline outer core plasmas, the collisionality is still large enough for a microtearing instability, even if other plasma parameters like $a / L_{T e}$ are kept constant.

\section{ROLE OF COLLISIONS}

Including collisions is essential for studying microtearing modes, since the associated resistivity enables magnetic reconnection. Moreover, one of the tearing mode drive mechanisms, the time derivative of the thermal force, requires finite collisionality. It is responsible for the energy transfer from the background temperature profile to the mode. Stability is thus expected at very low values of collisionality $\nu_{e i}$, where the thermal force vanishes. On the other hand, in the opposite limit of strong collisionality, the microtearing mode is also stabilized. Here the intuitive picture is that collisional decorrelation strongly inhibits electron motion along field lines, such that a current layer cannot be established. Early theoretical work showed that experimental conditions, which are typically between the collisionless and collisional limit, often can not be treated in a purely analytic fashion, even in slab geometry. $[15,16]$ (We note in passing that one important ingredient in these calculations is an appropriate energy dependence of the collision frequency $\nu_{e i}$.)

Further progress was achieved when Applegate and coworkers performed linear gyrokinetic simulations of microtearing modes in a systematic fashion.[22] As they also showed a few years ago, the energy dependence of $\nu_{e i}$ actually becomes less important when going from slab to toroidal geometry, and the qualitative behavior of the growth rate, peaking in the intermediate collisionality regime relevant to experiments is preserved. The latter finding is confirmed via GENE simulations presented in Fig. 8. Here, one observes that the growth rate changes only moderately over several orders of magnitude in $\nu_{e i}$. A maximum is found around collision frequencies at which the mean-free-path $\lambda_{\text {mfp }}$ approximately equals the connection length $2 \pi q R$, and it is 
more pronounced for larger mode numbers. Collisionality scales as $\nu_{e i} \sim n T^{-3 / 2}$ and is thus smaller in hotter plasmas. Our standard-tokamak parameter set is, however, still microtearing unstable when the collision frequency obtained for the ITER baseline scenario at $\rho_{\text {tor }} \sim 0.7-0.8$ is inserted, even if other plasma parameters like the temperature gradients are kept constant. Thus, the present work is also relevant to future tokamak devices.

\section{MICROTEARING MODE CURRENT LAYERS}

We now turn to a fundamental aspect of the physics behind the microtearing instability, which is the (temperature gradient driven) creation of a parallel current at a resonant flux surface, $q=m / n$. Interest arises, in particular, in the width of this current layer mainly for two reasons: (1) the comparison with analytic predictions yields insights into the underlying physics, and (2) the actual theoretical/numerical treatment can be validated. For example, the assumption of unmagnetized (adiabatic) ions only holds for $\Delta_{x} \ll \rho_{i}$, and the validity of this criterion can be tested. For illustration, a typical mode structure of the electron parallel current in the $x-z$ plane is shown in Fig. 9. One obvious difference with respect to existing theories is the peculiar parallel structure of $\tilde{j}_{\| e}$, which is linked to the strong ballooning behavior of $\tilde{A}_{\|}$.

Let us now briefly review certain aspects of the intuitive understanding of the linear physics involved. $[9,18]$ Standard microtearing theory assumes $k_{\|}=0$ at the rational flux surface (at $x=0$ ). There, electrons get accelerated by the parallel thermal force. This results in a parallel current which is bound to a region around the rational surface, where the inverse transit time is smaller than the mode frequency,

$$
v_{t e} k_{\|}(x) \lesssim \omega
$$

Here, $v_{t e}$ is the thermal electron velocity and $k_{\|}(x)$ is the $x$-dependent parallel wave number that vanishes right at the rational surface. In the opposite limit, when the transit time exceeds the mode frequency, the electrons will see an oscillating mode, which reduces their response in Ampère's law.

Now, in a sheared magnetic field, $k_{\|}(x)$ increases linearly with $x$. A geometric argument may be used to derive the simple formula $k_{\|}=k_{y} x / L_{s}$, where the magnetic shear length $L_{s}$ has been introduced. In toroidal geometry, the latter is given by $L_{s}=q R / \hat{s}$ (see, e.g., Refs. [18, 36]). Obviously, Eq. (1) does not include the effect of collisional broadening and is therefore only valid in the semicollisional to collisionless regime. Furthermore, the electrostatic potential $\tilde{\phi}$ has not been taken into account because electrostatic effects are assumed to be negligible for $x \ll \rho_{i}$. For larger current layers, the intuitive picture provided in Ref. [9] involves a stabilizing role of $\tilde{\phi}$, because the additional electric field $\tilde{E}_{\| \phi}=-\nabla \tilde{\phi}$ short-circuits the response $E_{\| A}=-(1 / c) \partial_{t} \tilde{A}_{\|}$. Another point of view is connected to the parallel force balance. When $k_{\|}$is significantly larger than zero, electrons are adiabatic and thus a current can only be generated in close proximity to the rational flux surface. However, Gladd and co-workers [16] pointed out the potential role of $\tilde{\phi}$ in destabilizing the current layer in the context of a semi-analytic theory, thereby further complicating the physical picture.

In the following, we will present linear gyrokinetic simulations with GENE in order to shed light on the impact of a wide range of plasma parameters on the microtearing current layer. Here, we will fix collisionality in the intermediate collisionality regime that is relevant for standard tokamaks. To compare with the intuitive picture, Eq. (1) is evaluated at the distance $x=\Delta_{x}$. The expression

$$
\frac{\Delta_{x}}{\rho_{i}}=\frac{q}{\hat{s}} \frac{\omega /\left(c_{s} / R\right)}{k_{y} \rho_{i}} \sqrt{\frac{m_{e}}{m_{i}}}
$$

characterizes the analytic prediction of the current layer width. The numerical eigenmodes computed with GENE are assessed by measuring the full width at half maximum of $j_{\|}(z=-\pi, x)$ at the torus inboard side. This position is chosen because we generally observe a widening at the outboard side. This structure actually corresponds to finite $k_{\|}$at $x=0$ and already gives some hint that the analytic model might be too simple. The particular structure of this widened mode generally depends on the plasma parameters, but the narrow layer at the inboard side appears to be universal. In these simulations, basically all the plasma parameters entering Eq. (2) have been varied. The frequency is actually not an input parameter, of course, but has been varied through the dependence on the electron temperature gradient, $\omega \sim a / L_{T e}$, which is, in fact, approximately linear. Our results suggest that some basic physics is indeed captured correctly by the intuitive model: The overall trends are generally reproduced. To give some examples, an increase in $q$, as well a decrease in $\omega$ or $\hat{s}$ widens the current layer, as expected. Quantitative agreement is only obtained in the sense that current layers tend to be as thin as $(0.1-0.5) \rho_{i}$, while the exact value can not be predicted by Eq. (2).

We have also investigated the impact of $\tilde{\phi}$ on our standard-tokamak microtearing mode, motivated by the above mentioned diverse effects and the gyrokinetic findings for the MAST spherical tokamak. In MAST, zeroing out $\tilde{\phi}$ is found to be stabilising, [22] suggesting that $\tilde{\phi}$ actively takes part in the instability mechanism. The red data points in Fig. 10 are obtained zeroing out $\tilde{\phi}$ for a $q$ scan in standard tokamak circular geometry. The modification of the growth rate or the current layer width is rather small. Measuring the mode growth, we find the trend that including $\tilde{\phi}$ is destabilizing for $q \lesssim 3$ and stabilizing for $q \gtrsim 3$. This is clear evidence for the existence of different flavors of the microtearing mode, an interesting aspect which remains to be better understood. 

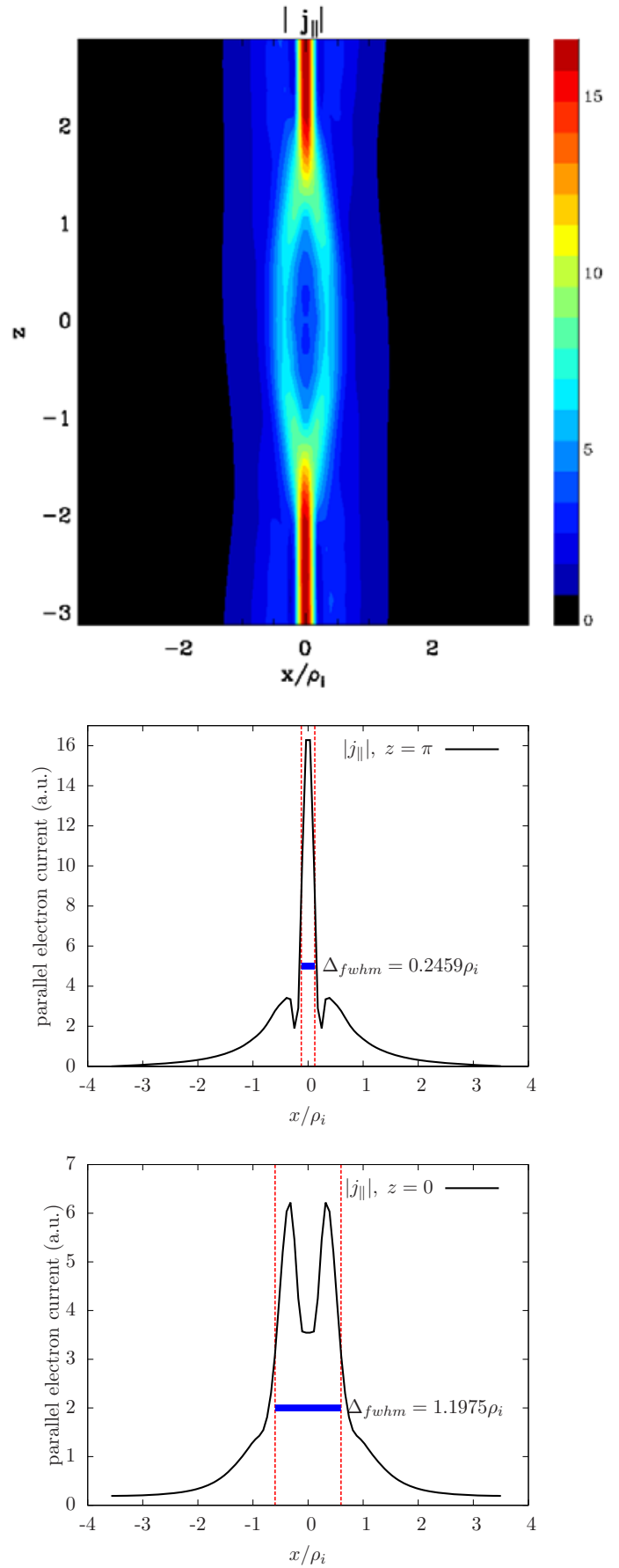

FIG. 9: (Color online) Typical structure of a current layer of a microtearing mode in the $x-z$ plane, taken at the torus inboard $(z=\pi)$ and outboard $(z=0)$ side.

\section{TURBULENT TRANSPORT DUE TO MICROTEARING MODES}

Elsewhere, we have recently reported the first nonlinear gyrokinetic microtearing simulations for standard tokamak plasmas. [3] In the following, we would like to briefly review some key results and discuss their relevance

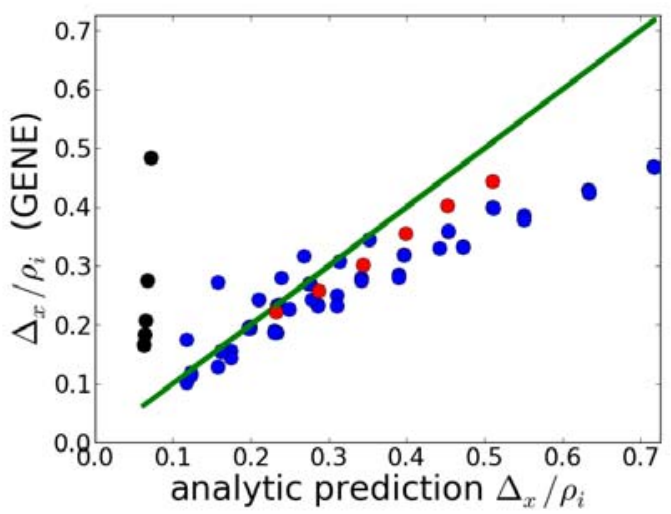

FIG. 10: (Color online) Comparison of the estimate in Eq. (2) for the current layer width to gyrokinetic simulation results, scanning essentially all of the parameters entering the analytic prediction. Blue dots vary $q, \hat{s}$ and $\omega$, red dots vary $q$ with $\tilde{\phi}=0$, and black dots vary $k_{y}$ in AUG geometry. The full width at half maximum of $\tilde{j}_{\| e}$, taken at the torus inboard side, is smaller than $\rho_{i}$. In the intermediate collisionality regime considered here, zeroing out $\tilde{\phi}$ has no significant influence.

TABLE II: Plasma parameters for $\rho_{\text {tor }}=0.5,0.65,0.8$ of AUG shot 26459 compared with the circular model used for the turbulence simulations.

\begin{tabular}{lrrrr}
\hline \hline & Circular & AUG 0.5 & AUG 0.65 & AUG 0.8 \\
\hline$x / a$ & 0.40 & 0.50 & 0.65 & 0.80 \\
$a / R$ & 0.37 & 0.37 & 0.37 & 0.37 \\
$a / L_{T e}$ & $0.9-2.2$ & 2.855 & 2.686 & 1.627 \\
$a / L_{T i}$ & 0.000 & 2.088 & 2.343 & 2.077 \\
$a / L_{n}$ & 0.370 & 0.341 & 0.365 & 0.303 \\
$T_{i} / T_{e}$ & 1.000 & 1.113 & 1.218 & 1.209 \\
$\nu_{e i} /\left(c_{s} / a\right)$ & 2.310 & 0.405 & 0.873 & 1.542 \\
$\beta[\%]$ & 0.600 & 0.586 & 0.363 & 0.251 \\
$q$ & 3.000 & 1.811 & 2.449 & 3.703 \\
$\hat{s}$ & 1.000 & 0.794 & 1.583 & 2.478 \\
$m_{i} / m_{p}$ & 1 & 2 & 2 & 2 \\
\hline \hline
\end{tabular}

for AUG and future standard tokamak experiments like ITER. The nominal parameters for the nonlinear GENE simulations are summarized in Tab. II (normalized to the minor radius and labelled as "Circular"). For comparison, the values for AUG discharge 26459 are shown. The value of $q$ and $\nu_{e i}$ fit the outer core regime, while the other parameters are close to those at the $\rho_{\text {tor }}=0.5$ surface. It is worth mentioning that increasing $q$ has been shown to increase the microtearing growth rate in this regime up to values of $q \sim 4.5$, above which increasing $q$ (and keeping all other parameters constant) is stabilizing. The ion temperature gradient has been set to zero for the nonlinear simulations to avoid multi-mode drive and to make the already demanding computations more tractable. It has been verified that the ion temperature gradient does not modify the microtearing branch, but 
FIG. 12: (Color online) Impact of an equilibrium $E \times B$ shear flow on microtearing turbulence. The saturated electron heat flux is reduced, but even with $E \times B$ shearing rates exceeding the linear growth rate by a factor of 10 , about $20 \%$ of the initial transport level remains.

it can be seen in Fig. 11 that the ITG branch disappeared. As a second simplification, an analytic model for the magnetic equilibrium, assuming concentric circular flux surfaces, is taken. This change also does not affect the microtearing mode substantially. We may thus expect that many qualitative and semi-quantitative features found in the nonlinear simulations will carry over to more realistic cases.

An interesting facet of microtearing turbulence which has not been addressed so far is the impact of an equilibrium $E \times B$ shear flow. A series of GENE simulations addressing this question are shown in Fig. 12. Here, the response of the saturated turbulent state (for the nominal parameters) to a number of different external $E \times B$ shearing rates is provided. Somewhat surprisingly, microtearing turbulence in our parameter regime seems to be only mildly susceptible to $E \times B$ shearing. This finding is in line with the observed weak dependence on nonlocal effects discussed above, and indicates that microtearing modes in standard tokamaks tend to be rather robust with respect to equilibrium shear flows.

Given this result, let us now return to simulations of microtearing turbulence without flow shear. Convergence studies indicate that a nominal resolution of $384 \times 64 \times$ $24 \times 32 \times 16$ grid points in $x \times y \times z \times v_{\|} \times \mu$ space is required. The domain width in the perpendicular plane is set by $l_{x}=150 \rho_{i}$ and $l_{y}=300 \rho_{i}$, resulting in a radial grid spacing of $\Delta_{x} \sim 0.5 \rho_{i}$. It has been verified that the linear growth rate is reasonably converged for all involved mode numbers. A typical resulting transport spectrum obtained by means of GENE is displayed in Fig. 11. While the particle flux is very low, one observes that the heat flux is dominated by the magnetic component $Q_{e}^{\mathrm{em}}$ in the electron channel, generally providing more than $80 \%$ of the total transport.

For the nonlinear saturation amplitude of the magnetic field fluctuations, the estimate

$$
\tilde{B}_{x} / B_{0} \sim \rho_{e} / L_{T e}
$$

has been derived in an early semi-analytic work by Drake and co-workers. [25] Here, $\rho_{e}$ is the electron Larmor radius. We find that this relation generally describes the GENE simulation data fairly well. Varying $a / L_{T e}$, both in the scaling of the magnetic field fluctuations with $a / L_{T e}$ and their magnitude is within some scatter described by Eq. (3). It must also be pointed out, however, that Eq. (3) has clear limits. This estimate fails to capture both the threshold behavior in $\beta_{e}$ and $a / L_{T e}$ as well as the dependencies on other parameters, like collisionality (see, e.g., Fig. 8), or geometric quantities like $q$. In this context, it is important to consider that the simulations have been performed somewhat above the linear threshold, and that a large influence of $\beta_{e}$ is indeed found. The derivation of the Drake scaling involves a balance of linear drive and nonlinear transfer to small $k_{y}$. This physical picture has to be refined, however. On the basis of our nonlinear gyrokinetic simulations and several supporting linear growth rate studies, Eq. (3) can only be retained by balancing low- $k_{y}$ linear drive and high- $k_{y}$ dissipation. This new view is motivated by measuring free energy sources and sinks in the simulation data, exhibiting an energy transfer to smaller scales in the perpendicular plane.[3]

As mentioned before, the thermal transport associated with microtearing turbulence is dominated by the magnetic contribution in the electron channel. The corresponding electron heat diffusivity $\chi_{e}^{\mathrm{em}}$ is given in terms of the electron heat flux $Q_{e}^{\mathrm{em}}$ via

$$
Q_{e}^{\mathrm{em}}=-n_{e} \chi_{e}^{\mathrm{em}} \nabla T_{e} .
$$

This diffusivity, as obtained from a set of about 15 GENE simulations (varying the electron temperature gradient 
FIG. 14: (Color online) Magnetic diffusivity computed from Gene data with Eq. (6). The quasilinear result $D_{M}=$ $\pi q R\left(\tilde{B}_{x} / B_{0}\right)^{2}$ is well applicable in the present regime. We find two outliers at small amplitude, where stochasticity is not established.
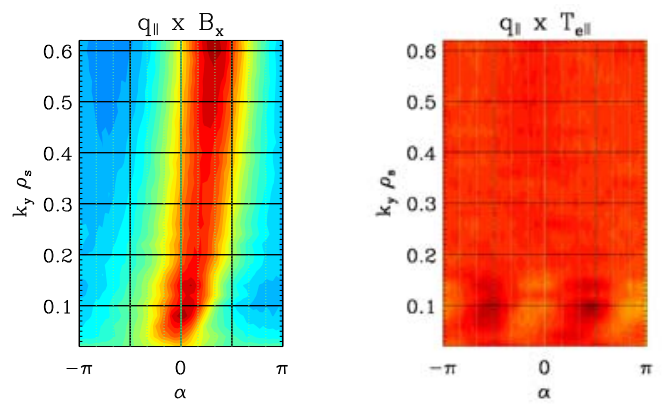

FIG. 15: (Color online) Cross phase analyses in the strongly driven microtearing turbulence. In the region of transport peak at $k_{y} \rho_{i} \sim 0.2, \tilde{q}_{\|}$and $\tilde{B}_{x}$ are in phase (left) while $\tilde{q}_{\|}$and $\tilde{T}_{\|}$have random phase (right). In conclusion, $\mathcal{T}_{3}$ dominates over $\mathcal{T}_{1}$, and Eq. (5) is applicable.

strongly driven microtearing turbulence. For the latter cases, we find the magnetic diffusivity to be well characterized by the quasilinear result $D_{M}=L_{C}\left(\tilde{B}_{x} / B_{0}\right)^{2}$, with the auto-correlation length taken as $L_{C}=\pi q R=$ $\pi \Delta k_{\|}^{-1}$ and the parallel spectral width $\Delta k_{\|} \cdot$ [40]. This correlation length can be modified by collisional decorrelation when the electron mean free path $\lambda_{\mathrm{mfp}}=v_{t e} / \nu_{e i}$ becomes small. Following Ref. [41], we introduce an effective length $L_{\text {eff }}=\pi\left((q R)^{-1}+\lambda_{\mathrm{mfp}}^{-1}\right)=0.94 L_{C}$ in our case. Our results for stochastic heat transport are in good agreement with the heat flux expression $Q_{e}^{\text {st }}=\sqrt{2 / \pi} f_{p} v_{t e} D_{M} n \partial T / \partial r$ of Ref. [6]. This is valid for small particle flux and refines slab geometry results of Ref. [41] by taking into account the passing particle fraction $f_{p} \approx 1-\sqrt{r / R}$. The corresponding heat diffusivity (including the small collisional correction) is given as $\chi_{e}^{\text {st }}=\sqrt{2 / \pi} f_{p} v_{t e} L_{\text {eff }} / L_{C} D_{M}$. This yields $\eta=1.45$, about $5 \%$ above our fit on the simulated data in Fig. 13. 

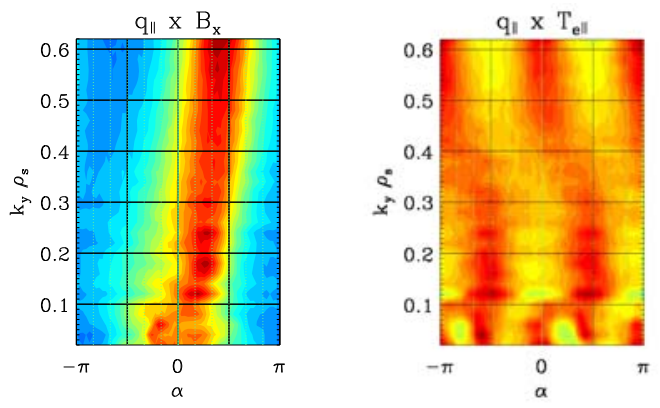

FIG. 16: (Color online) In weakly driven cases, the cross phase corresponding to $\mathcal{T}_{3}$ (left) is less pronounced at the transport peak $k_{y} \rho_{i} \lesssim 0.1$. Also, $\tilde{q}_{e \|}$ is partially in phase with $\tilde{T}_{e \|}$, as seen in the right panel. One can thus expect modifications to the Rechester-Rosenbluth model, Eq. (5).

One can also take another point of view, writing the radial electron heat flux as

$$
Q_{e}^{\mathrm{em}}=\left\langle\tilde{q}_{e \|} \tilde{B}_{x}\right\rangle / B_{0}
$$

with

$$
\tilde{q}_{e \|}=-n_{e 0} \chi_{e \|}\left(\frac{\mathrm{d} \tilde{T}_{e \|}}{\mathrm{d} z}+\frac{\tilde{B}_{x}}{B_{0}} \frac{\mathrm{d} \tilde{T}_{e \|}}{\mathrm{d} x}+\frac{\tilde{B}_{x}}{B_{0}} \frac{\mathrm{d} T_{e 0}}{\mathrm{~d} x}\right) .
$$

The three terms on the right-hand side denote the components of $\nabla_{\|} T$ along the perturbed magnetic field lines. We refer to them as $\mathcal{T}_{1}, \mathcal{T}_{2}$, and $\mathcal{T}_{3}$ in the following. The parallel conductivity has been computed for slab geometry as $\chi_{e \|}=9 /(5 \sqrt{\pi})\left(v_{t e} / k_{\|}\right)$in the adiabatic limit.[42] In our toroidal model with quasiperiodic boundary conditions along the field line, technically there is no quantity like $k_{\|}$, but we may estimate $k_{\|} \sim 1 /(q R)$. Doing this, one obtains Eq. (5), provided $\mathcal{T}_{3}$ dominates.

Indeed, the dominant role of $\mathcal{T}_{3}$ can be confirmed by observing cross phase relations between the fluctuating quantities shown in Figs. 15 and 16. In typical strong microtearing turbulence cases, one concludes $\mathcal{T}_{3} \gg \mathcal{T}_{1}$ from the fact that $\tilde{q}_{\|}$and $\tilde{T}_{\|}$have random phases and a tendency to anticorrelate at a phase of $\pi / 2$, while $\tilde{q}_{\|}$ and $\tilde{B}_{x}$ are correlated around the transport peak in $k_{y}$. In weakly turbulent cases, the correlation of $\tilde{q}_{\|}$and $\tilde{B}_{x}$ is less pronounced, in particular, at the transport peak $k_{y} \sim 0.06$, and at the same time, $\tilde{q}_{\|}$and $\tilde{T}_{\|}$are partially correlated. One can conclude that in these weakly driven cases, Eq. (5) is not applicable and, as a result, the diffusivity is overpredicted. The second term, $\mathcal{T}_{2}$, may also contribute, in principle. We find that $\tilde{T}_{\|}$and $\tilde{B}_{x}$ (and thus $\tilde{q}_{\|}$and $\tilde{T}_{\|} \tilde{B}_{x}$ ) are in phase (between $-\pi / 8$ and $\pi / 4$ ) in both cases.

In the present context, we would like to note that the application of diffusivity models may also be restricted by completely different physics. Del Castillo-Negrete [43] has shown that the electron heat flux along perturbed magnetic field lines can, in general, be non-diffusive.
Nonlocal effects (in the parallel direction) actually prohibit the description of the radial heat flux in terms of a simple diffusivity. In the present gyrokinetic simulations, no such non-diffusive behavior has been found, however.

Finally, we would like to address the question if the resulting transport levels are sufficiently large to be considered experimentally relevant. To assess this issue, we use the plasma parameters corresponding to the AUG discharge 26459. These include $R=1.65 \mathrm{~m}, B_{0}=2.37$ $\mathrm{T}, T_{e}=1.09 \mathrm{keV}, n_{e}=7.94 \times 10^{19} \mathrm{~m}^{-3}$, and $\rho^{*}=0.003$. For an electron temperature gradient of $a / L_{T e}=1.85$ $\left(R / L_{T e}=5\right)$ and the otherwise nominal parameters summarized in Tab. II, we find a magnetic perturbation level of $\tilde{B}_{x} / B_{0} \sim 3.5 \cdot 10^{-4}$ and a corresponding electron heat diffusivity of $\chi_{e}^{\mathrm{em}} \sim 1.4 \mathrm{~m}^{2} / \mathrm{s}$. These numbers suggest that microtearing turbulence must be considered an additional candiate for explaining the observed anomalous heat losses in present-day and future (standard) tokamaks.

\section{SUMMARY AND CONCLUSIONS}

In summary, our work - based on comprehensive gyrokinetic simulations - provides strong evidence for the existence of microtearing turbulence in standard tokamak devices. In particular, using realistic equilibria and experimentally measured temperature and density profiles, microtearing modes have been observed at various mode rational surfaces in the outer core of AUG discharge 26459. A study of system size effects was performed by variation of the parameter $\rho^{*}$ in a circular geometry model. The finite temperature gradient profile width which is known to substantially weaken ITG modes was shown to be less effective for microtearing modes. The growth rate obtained in global simulations stays close to that in the local limit even for narrow temperature gradient profiles, strongly supporting a local treatment of the microtearing problem. In addition, the microtearing mode is found to be only weakly affected by background $E \times B$ shear flows. Therefore, in the remainder of this work, nonlocal and equilibrium shear effects have been neglected.

In terms of linear physics, several peculiar features of microtearing modes in standard tokamaks have been observed. Interestingly, their growth rate spectrum tends to peak at rather low binormal wavenumbers of $k_{y} \rho_{s} \sim 0.1$, which allows them to co-exist with ITG/TEM modes at larger wavenumbers. The most critical plasma parameters turn out to be $\beta_{e}$ and $a / L_{T e}$, with the linear threshold of the latter typically below the respective value for ITG modes. In addition, collisions play an important role. Our gyrokinetic simulations exhibit only a very moderate dependence on the collision frequency, such that microtearing modes are also expected to appear in low-collisionality plasmas like those in ITER. Overall, the linear physics of microtearing modes remains to be bet- 
ter understood, however, as was exemplified by a study on the width and parallel structure of the current layer where common models cannot always capture the observed trends.

In nonlinear simulations, one finds that the heat transport is dominated by the magnetic component in the electron channel, which can be well described by means of a Rechester-Rosenbluth model. Moreover, it was found that the Chirikov criterion for island overlap implies the existence of a threshold in the drive strength, and therefore a nonlinear up-shift of the critical electrontemperature gradient. An analogy to the well-known Dimits-shift for ITG modes can be seen here, although the underlying physics is completely different. Inserting the plasma parameters of an actual AUG discharge (shot 26459), one obtains electron heat diffusivities of up to a few $\mathrm{m}^{2} / \mathrm{s}$, depending mainly on the precise value of the electron temperature gradient. Consequently, the present work points towards a role of microtearing turbulence in standard tokamaks, including ITER.

\section{Acknowledgements}

We gratefully acknowledge fruitful discussions with K. Lackner, F. Merz, W. M. Nevins, W. Guttenfelder, J. E. Boom, and E. Wolfrum. Moreover, we would like to thank P. Schneider and M. Dunne for support with obtaining the AUG geometry reconstruction and kinetic profiles. The simulation results were obtained with the help of the high performance computing resources provided by the RZG in Garching, Germany and on the HPC-FF system at Forschungszentrum Jülich, Germany.
[1] http://www.iter.org, 2011.

[2] F. Jenko, W. Dorland, M. Kotschenreuther, and B. N. Rogers. Electron temperature gradient driven turbulence. Phys. Plasmas, 7:1904-1910, May 2000.

[3] H. Doerk, F. Jenko, M. J. Pueschel, and D. R. Hatch. Gyrokinetic microtearing turbulence. Phys. Rev. Lett, 106(15):155003, April 2011.

[4] W. Guttenfelder, J. Candy, S. M. Kaye, W. M. Nevins, E. Wang, R. E. Bell, G. W. Hammett, B. P. Leblanc, D. R. Mikkelsen, and H. Yuh. Electromagnetic transport from microtearing mode turbulence. Phys. Rev. Lett, 106(15):155004, April 2011.

[5] D. R. Hatch, M. J. Pueschel, W. M. Nevins, F. Jenko, P. W. Terry, and H. Doerk. Origin of Magnetic Stochasticity and Transport in Plasma Microturbulence. Phys. Rev. Lett, 108(23):235002, January 2012.

[6] W. M. Nevins, E. Wang, and J. Candy. Magnetic stochasticity in gyrokinetic simulations of plasma microturbulence. Phys. Rev. Lett, 106(6):065003, February 2011.

[7] T. H. Stix. Magnetic braiding in a toroidal plasma. Phys. Rev. Lett, 30:833-835, April 1973.

[8] R. D. Hazeltine, D. Dobrott, and T. S. Wang. Kinetic theory of tearing instability. Phys. Fluids, 18:1778-1786, December 1975.

[9] J. F. Drake and Y. C. Lee. Kinetic theory of tearing instabilities. Phys. Fluids, 20:1341-1353, August 1977.

[10] L. Chen, P. H. Rutherford, and W. M. Tang. Driftmodified tearing instabilities due to trapped electrons. Phys. Rev. Lett, 39:460-463, August 1977.

[11] J. D. Callen. Drift-wave turbulence effects on magnetic structure and plasma transport in tokamaks. Phys. Rev. Lett, 39:1540-1543, December 1977.

[12] M. J. Pueschel, M. Kammerer, and F. Jenko. Gyrokinetic turbulence simulations at high plasma beta. Phys. Plasmas, 15(10):102310, October 2008.

[13] A. B. Hassam. Fluid theory of tearing instabilities. Phys. Fluids, 23:2493-2497, December 1980.

[14] A. B. Hassam. Higher-order chapman-enskog theory for electrons. Phys. Fluids, 23:38-43, January 1980.

[15] D. A. Dippolito, Y. C. Lee, and J. F. Drake. Linear stabil- ity of high-m drift-tearing modes. Phys. Fluids, 23:771782, April 1980.

[16] N. T. Gladd, J. F. Drake, C. L. Chang, and C. S. Liu. Electron temperature gradient driven microtearing mode. Phys. Fluids, 23:1182-1192, June 1980.

[17] P. J. Catto and M. N. Rosenbluth. Trapped electron modifications to tearing modes in the low collision frequency limit. Phys. Fluids, 24:243-255, February 1981.

[18] J. W. Connor, S. C. Cowley, and R. J. Hastie. Microtearing stability in tokamaks. Plasma Phsy. Controlled Fusion, 32:799-817, October 1990.

[19] M. Kotschenreuther, W. Dorland, Q. P. Liu, M. C. Zarnstorff, R. L. Miller, and Y. R. Lin-Liu. Attaining neoclassical transport in ignited tokamaks. Nucl. Fusion, 40:677-684, March 2000.

[20] D. J. Applegate, C. M. Roach, S. C. Cowley, W. D. Dorland, N. Joiner, R. J. Akers, N. J. Conway, A. R. Field, A. Patel, M. Valovic, and M. J. Walsh. Microstability in a "mast-like" high confinement mode spherical tokamak equilibrium. Phys. Plasmas, 11:5085-5094, November 2004.

[21] C. M. Roach, D. J. Applegate, J. W. Connor, S. C. Cowley, W. D. Dorland, R. J. Hastie, N. Joiner, S. Saarelma, A. A. Schekochihin, R. J. Akers, C. Brickley, A. R. Field, M. Valovic, and the MAST Team. Microstability physics as illuminated in the spherical tokamak. Plasma Phsy. Controlled Fusion, 47:B323-B336, December 2005.

[22] D J Applegate, C M Roach, J W Connor, S C Cowley, W Dorland, R J Hastie, and N Joiner. Micro-tearing modes in the mega ampere spherical tokamak. Plasma Phsy. Controlled Fusion, 49(8):1113-1128, 2007.

[23] L. Vermare, C. Angioni, A. Bottino, A. G. Peeters, and ASDEX Upgrade Team. $\beta$ dependence of microinstabilities using linear gyrokinetic simulations. J. Phys. Conference Series, 123(1):012040, July 2008.

[24] D. Told, F. Jenko, P. Xanthopoulos, L. D. Horton, E. Wolfrum, and ASDEX Upgrade Team. Gyrokinetic microinstabilities in asdex upgrade edge plasmas. Phys. Plasmas, 15(10):102306, October 2008.

[25] J. F. Drake, N. T. Gladd, C. S. Liu, and C. L. Chang. Microtearing modes and anomalous transport in tokamaks. 
Phys. Rev. Lett, 44:994-997, April 1980.

[26] K. L. Wong, S. Kaye, D. R. Mikkelsen, J. A. Krommes, K. Hill, R. Bell, and B. Leblanc. Microtearing instabilities and electron transport in the nstx spherical tokamak. Phys. Rev. Lett, 99(13):135003, September 2007.

[27] W. Guttenfelder,J. Candy, S. M. Kaye, W. M. Nevins, E. Wang, J. Zhang, R. E. Bell1, N. A. Crocker, G. W. Hammett, B. P. LeBlanc, D. R. Mikkelsen, Y. Ren, and H. Yuh Simulation of microtearing turbulence in national spherical torus experiment Phys. Plasmas, 19(5):056119, May 2012.

[28] T. Görler, X. Lapillonne, S. Brunner, J. Chowdhury, T. Dannert, F. Jenko, B. F. McMillan, F. Merz, D. Told, and L. Villard. Nonlocal effects in gyrokinetic turbulence simulations using GENE. J. Phys. Conference Series, 260(1):012011, November 2010.

[29] T. Görler, X. Lapillonne, S. Brunner, T. Dannert, F. Jenko, F. Merz, and D. Told. The global version of the gyrokinetic turbulence code GENE. J. Comput. Phys., 230:7053-7071, August 2011.

[30] B. F. McMillan, X. Lapillonne, S. Brunner, L. Villard, S. Jolliet, A. Bottino, T. Görler, and F. Jenko. System size effects on gyrokinetic turbulence. Phys. Rev. Lett, 105(15):155001, October 2010.

[31] F. Merz. Gyrokinetic Simulation of Multimode Plasma Turbulence. $\mathrm{PhD}$ thesis, Universität Münster, 2009.

[32] P. Xanthopoulos, D. Mikkelsen, F. Jenko, W. Dorland, and O. Kalentev. Verification and application of numerically generated magnetic coordinate systems in gyrokinetics. Phys. Plasmas, 15(12):122108, December 2008.

[33] J. Boom, E. Wolfrum, I. G. J. Classen, P. C. de Vries, M. Maraschek, W. Suttrop, C. P. Pérez von Thun, A. J. H. Donné, B. J. Tobias, C. W. Domier, N. C. Luhmann Jr., H. K. Park, and the ASDEX Upgrade Team. 2D characterization of type-II edge localized modes at ASDEX Upgrade. accepted for publication in Nucl. Fusion

[34] E. Wolfrum, M. Bernert, J. E. Boom, A. Burckhart, I. G. J. Classen, G. D. Conway, T. Eich, R. Fischer, A. Gude, A. Herrmann, N. C. Luhmann, Jr., M. Maraschek, R. McDermott, H. K. Park, T. Pütterich,
J. Vicente, B. Wieland, M. Willensdorfer, and the ASDEX Upgrade Team. Characterization of edge profiles and fluctuations in discharges with type-ii and nitrogenmitigated edge localized modes in asdex upgrade. Plasma Phsy. Controlled Fusion, 53(8):085026, August 2011.

[35] D. R. Smith, W. Guttenfelder, B. P. LeBlanc, and D. R. Mikkelsen. Identification of microtearing modes below the ion gyroscale in the national spherical torus experiment. Plasma Phsy. Controlled Fusion, 53(3):035013, March 2011.

[36] John Wesson. Tokamaks. Clarendon Press - Oxford, 3 edition, 2004.

[37] A. B. Rechester and M. N. Rosenbluth. Electron heat transport in a tokamak with destroyed magnetic surfaces. Phys. Rev. Lett, 40:38-41, January 1978.

[38] B. V. Chirikov. A universal instability of manydimensional oscillator systems. Phys. Rep., 52:263-379, May 1979.

[39] A. M. Dimits, G. Bateman, M. A. Beer, B. I. Cohen, W. Dorland, G. W. Hammett, C. Kim, J. E. Kinsey, M. Kotschenreuther, A. H. Kritz, L. L. Lao, J. Mandrekas, W. M. Nevins, S. E. Parker, A. J. Redd, D. E. Shumaker, R. Sydora, and J. Weiland. Comparisons and physics basis of tokamak transport models and turbulence simulations. Phys. Plasmas, 7:969-983, March 2000.

[40] J. A. Krommes, C. Oberman, and R. G. Kleva. Plasma transport in stochastic magnetic fields. part 3. kinetics of test particle diffusion. J. Plasma Phys., 30:11, 1983.

[41] R. W. Harvey, M. G. McCoy, J. Y. Hsu, and A. A. Mirin. Electron dynamics associated with stochastic magnetic and ambipolar electric fields. Phys. Rev. Lett, 47:102105, July 1981.

[42] Z. Chang and J. D. Callen. Unified fluid/kinetic description of plasma microinstabilities. i - basic equations in a sheared slab geometry. ii - applications. Phys. Fluids B, 4:1167-1181, May 1992.

[43] D. Del-Castillo-Negrete and L. Chacón. Local and nonlocal parallel heat transport in general magnetic fields. Phys. Rev. Lett, 106(19):195004, May 2011. 